\title{
Quantum chaos and chiral symmetry at the QCD and QED phase transition
}

\author{
Elmar Bittner, Harald Markum, and Rainer Pullirsch ${ }^{\mathrm{a}}$ \\ anstitute for Nuclear Physics, TU-Wien, Wiedner Hauptstraße 8-10, A-1040 Vienna, Austria
}

\begin{abstract}
We investigate the eigenvalue spectrum of the staggered Dirac matrix in SU(3) gauge theory and in full QCD as well as in quenched U(1) theory. As a measure of the fluctuation properties of the eigenvalues, we consider the nearest-neighbor spacing distribution. We find that in all regions of their phase diagrams, compact lattice gauge theories have bulk spectral correlations given by random matrix theory, which is an indication for quantum chaos. In the confinement phase, the low-lying Dirac spectrum of these quantum field theories is well described by random matrix theory, exhibiting universal behavior. Related results for gauge theories with minimal coupling are now discussed also in the chirally symmetric phase.
\end{abstract}

\section{Quantum chaos}

The eigenvalues of the Dirac operator are of great interest for the universality of important features of QCD and QED. On the one hand, the accumulation of small eigenvalues is, via the Banks-Casher formula [1], related to the spontaneous breaking of chiral symmetry. On the other hand, the fluctuation properties of the eigenvalues in the bulk of the spectrum can be described by random matrix theory (RMT), see Ref. [2]. For example, the nearest-neighbor spacing distribution $P(s)$, i.e., the distribution of spacings $s$ between adjacent eigenvalues on the unfolded scale, agrees with the Wigner surmise of RMT. According to the Bohigas-Giannoni-Schmit conjecture [3], quantum systems whose classical counterparts are chaotic [4] have a nearest-neighbor spacing distribution given by RMT whereas systems whose classical counterparts are integrable obey a Poisson distribution, $P_{\mathrm{P}}(s)=e^{-s}$. Therefore, the specific form of $P(s)$ is often taken as a criterion for the presence or absence of "quantum chaos".

In RMT, one has to distinguish several universality classes which are determined by the symmetries of the system. For the case of the QCD Dirac operator, this classification was done in Ref. [5]. Depending on the number of colors and the representation of the quarks, the Dirac operator is described by one of the three chiral ensembles of
RMT. As far as the fluctuation properties in the bulk of the spectrum are concerned, the predictions of the chiral ensembles are identical to those of the ordinary ensembles [6]. In Ref. [2], the Dirac matrix was studied for color-SU(2) using both staggered and Wilson fermions which correspond to the chiral symplectic (chSE) and orthogonal (chOE) ensemble, respectively. Here [7], we study $\mathrm{SU}(3)$ with staggered fermions which corresponds to the chiral unitary ensemble (chUE). The RMT result for the nearest-neighbor spacing distribution can be expressed in terms of so-called prolate spheroidal functions, see Ref. [8]. A very good approximation to $P(s)$ is provided by the Wigner surmise for the unitary ensemble,

$P_{\mathrm{W}}(s)=\frac{32}{\pi^{2}} s^{2} e^{-4 s^{2} / \pi}$.

We generated gauge field configurations using the standard Wilson plaquette action for $\mathrm{SU}(3)$ with and without dynamical fermions in the Kogut-Susskind prescription. We have worked on a $6^{3} \times 4$ lattice with various values of the inverse gauge coupling $\beta=6 / g^{2}$ both in the confinement and deconfinement phase. We typically produced 10 independent equilibrium configurations for each $\beta$.

The Dirac operator, $\not D=\not D+i g \not$, is antiHermitian so that the eigenvalues $\lambda_{n}$ of $i \not D$ are real. Because of $\left\{\not D, \gamma_{5}\right\}=0$ the nonzero $\lambda_{n}$ occur in pairs of opposite sign. All spectra were checked 

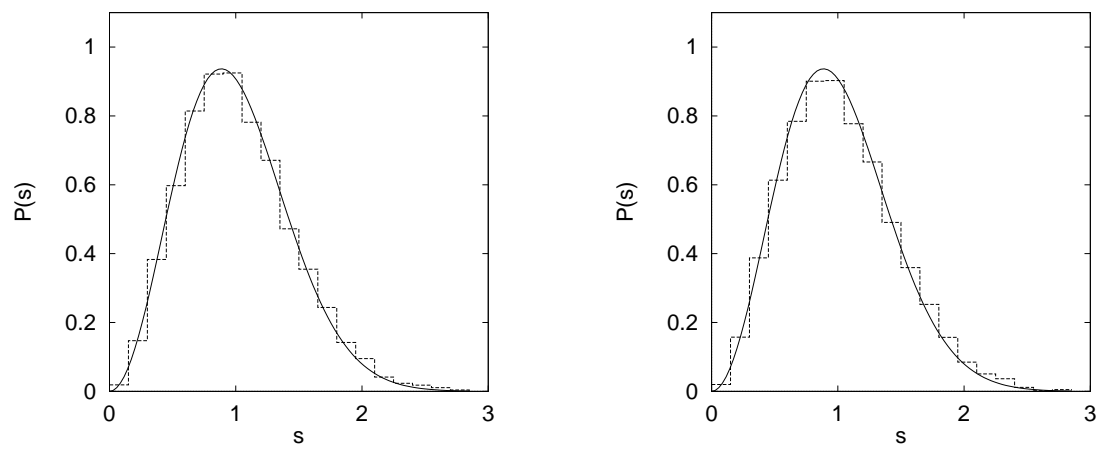

Figure 1. Nearest-neighbor spacing distribution $P(s)$ for full QCD on a $6^{3} \times 4$ lattice in the confinement phase (left) and in the deconfinement phase (right) compared with the random matrix result (solid lines). There are no changes in $P(s)$ across the deconfinement phase transition.
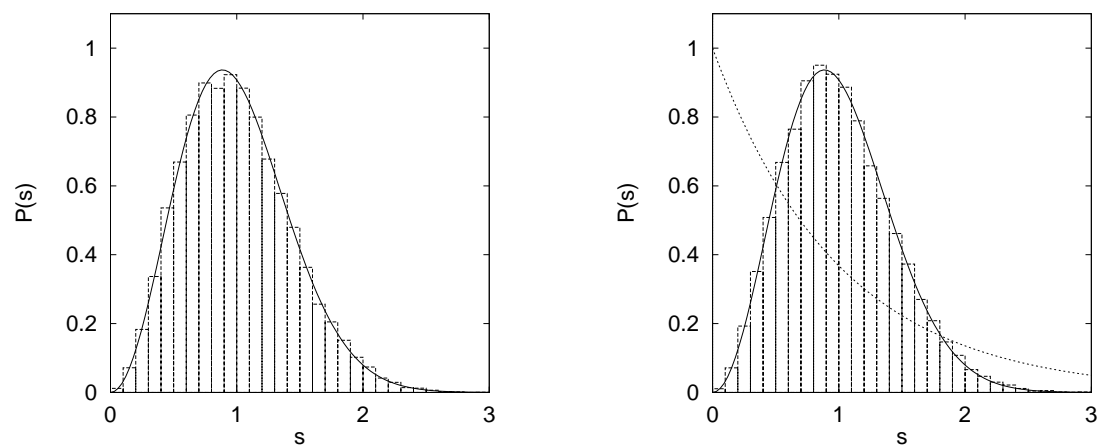

Figure 2. Nearest-neighbor spacing distribution $P(s)$ for $\mathrm{U}(1)$ gauge theory on an $8^{3} \times 6$ lattice in the confined phase (left) and in the Coulomb phase (right). The theoretical curves are the chUE result, Eq. (1), and the Poisson distribution, $P_{\mathrm{P}}(s)=e^{-s}$.

against the analytical sum rules $\sum_{n} \lambda_{n}=0$ and $\sum_{\lambda_{n}>0} \lambda_{n}^{2}=3 V$, where $\mathrm{V}$ is the lattice volume. To construct the nearest-neighbor spacing distribution from the eigenvalues, one first has to "unfold" the spectra [8].

Figure 1 compares $P(s)$ of full QCD with $N_{f}=$ 3 flavors and quark mass $m a=0.05$ to the RMT result. In the confinement $(\beta=5.2)$ as well as in the deconfinement $(\beta=5.4)$ phase we observe agreement with RMT up to very high $\beta$ (not shown). The observation that $P(s)$ is not influenced by the presence of dynamical quarks is expected from the results of Ref. [6], which apply to the case of massless quarks. Our results, and those of Ref. [2], indicate that massive dynamical quarks do not affect $P(s)$ either.

We have also investigated the staggered Dirac spectrum of $4 \mathrm{~d} \mathrm{U}(1)$ gauge theory which corresponds to the chUE of RMT. At $\beta_{c} \approx 1.01 \mathrm{U}(1)$ gauge theory undergoes a phase transition between a confinement phase with mass gap and monopole excitations for $\beta<\beta_{c}$ and the Coulomb phase which exhibits a massless photon for $\beta>$ $\beta_{c}$ [9. As for $\mathrm{SU}(2)$ and $\mathrm{SU}(3)$ gauge groups, we expect the confined phase to be described by RMT, whereas free fermions yield the Poisson distribution. The question arose whether the Coulomb phase would be described by RMT or by the Poisson distribution [10]. The nearestneighbor spacing distributions for an $8^{3} \times 6$ lattice at $\beta=0.9$ (confined phase) and at $\beta=1.1$ (Coulomb phase), averaged over 20 independent configurations, are depicted in Fig. 2. Both are well described by the chUE of RMT. 
No signs for a transition to Poisson regularity are found. The deconfinement phase transition does not seem to coincide with a transition in the spacing distribution. For very large values of $\beta$ far into the deconfinement region, the eigenvalues start to approach the degenerate eigenvalues of the free theory, given by $\lambda^{2}=$ $\sum_{\mu=1}^{4} \sin ^{2}\left(2 \pi n_{\mu} / L_{\mu}\right) / a^{2}$, where $a$ is the lattice constant, $L_{\mu}$ is the number of lattice sites in the $\mu$-direction, and $n_{\mu}=0, \ldots, L_{\mu}-1$. In this case, the spacing distribution is neither Wigner nor Poisson. It is possible to lift the degeneracies of the free eigenvalues using an asymmetric lattice where $L_{x}, L_{y}$, etc. are relative primes and, for large lattices, the distribution is then Poisson, $P_{\mathrm{P}}(s)=e^{-s}$, see Fig. 3.

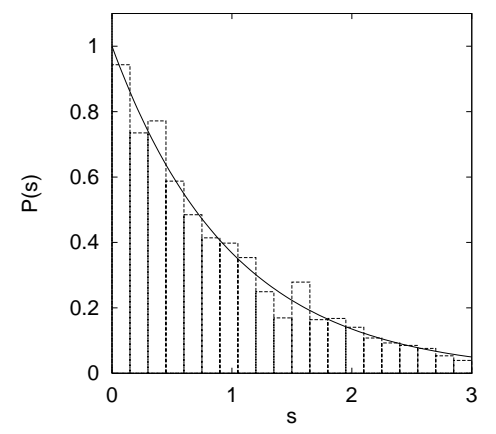

Figure 3. Nearest-neighbor spacing distribution $P(s)$ for the free Dirac operator on a $53 \times 47 \times 43 \times$ 41 lattice compared with a Poisson distribution.

\section{Chiral symmetry}

We have continued our investigations with a study of the distribution of the small eigenvalues in the whole phase diagram. The BanksCasher formula [1] relates the Dirac eigenvalue density $\rho(\lambda)$ at $\lambda=0$ to the chiral condensate, $\Sigma \equiv|\langle\bar{\psi} \psi\rangle|=\lim _{\varepsilon \rightarrow 0} \lim _{V \rightarrow \infty} \pi \rho(\varepsilon) / V$. The microscopic spectral density, $\rho_{s}(z)=$ $\lim _{V \rightarrow \infty} \rho(z / V \Sigma) / V \Sigma$, should be given by the appropriate prediction of RMT [11], which also generates the Leutwyler-Smilga sum rules [12].

We present results in Fig. 1 for $\mathrm{SU}(3)$ theory and the staggered Dirac operator on a $4^{4}$ lattice from 5000 configurations for $\beta=5.4$ and 3000 configurations for $\beta=5.6$ and $\beta=5.8$ around the critical temperature $\beta_{c} \approx 5.7$. In the confinement phase, both the microscopic spectral density $\rho_{s}(z)$ and the distribution $P\left(\lambda_{\min }\right)$ of the smallest eigenvalue agree with the RMT predictions of the chUE for topological charge $\nu=0$ [13].

Our analog presentation for $\mathrm{U}(1)$ theory is from 10000 configurations on a $4^{4}$ lattice around the critical coupling. In the left plot of Fig. 5 a comparison with RMT for the microscopic spectral density $\rho_{s}(z)$ yields again quite satisfactory agreement in the confinement. The analytical RMT result for the (quenched) chUE and $\nu=0$ is given by [11] $\rho_{s}(z)=z\left[J_{0}^{2}(z)+J_{1}^{2}(z)\right] / 2$, where $J$ denotes the Bessel function. The chiral condensate $\Sigma$ can be obtained by extrapolating the histogram for $\rho(\lambda)$ to $\lambda=0$ and using the Banks-Casher relation [14]. The right plot in Fig. 5 exhibits the distribution $P\left(\lambda_{\min }\right)$ of the smallest eigenvalue, being in the chirally broken phase in accordance with the prediction of the (quenched) chUE of RMT for topological charge $\nu=0$, $P\left(\lambda_{\min }\right)=(V \Sigma)^{2}\left(\lambda_{\min } / 2\right) \exp \left(-\left(V \Sigma \lambda_{\min }\right)^{2} / 4\right)$.

The quasi-zero modes which are responsible for the chiral condensate $\Sigma \neq 0$ build up when we cross from the deconfinement into the confined phase. Figures 1 and . demonstrate that both $\rho(\lambda)$ and $P\left(\lambda_{\text {min }}\right)$ plotted with varying $\beta$ on identical scales, respectively, can serve as an indicator for the phase transition.

In Fig. 6 we turn to a discussion of the spectrum in the quark-gluon plasma and Coulomb phase. From RMT a functional form of $\rho(\lambda)=$ $C\left(\lambda-\lambda_{0}\right)^{2 m+1 / 2}$ is expected at the onset of the eigenvalue density [15]. A fit to the data in the regime up to $\lambda=1$ yields $m=0.081$ (1) for the non-Abelian and $m=0.084(1)$ for the Abelian theory, in agreement with recent studies [16]. This suggests that both theories correspond to universality class $m=0$. For this class a microscopic level density involving the Airy function can be deduced from RMT [17]. A rescaling of our data from the $4^{4}$ lattice to this functional form is not satisfactory for both theories [16]. The reason might be (i) that the lowest eigenvalue is still influenced by quasi-zero modes from the confinement and (ii) that already the first eigenvalues lie above an analogue of the Thouless energy. With increasing lattice size these ef- 

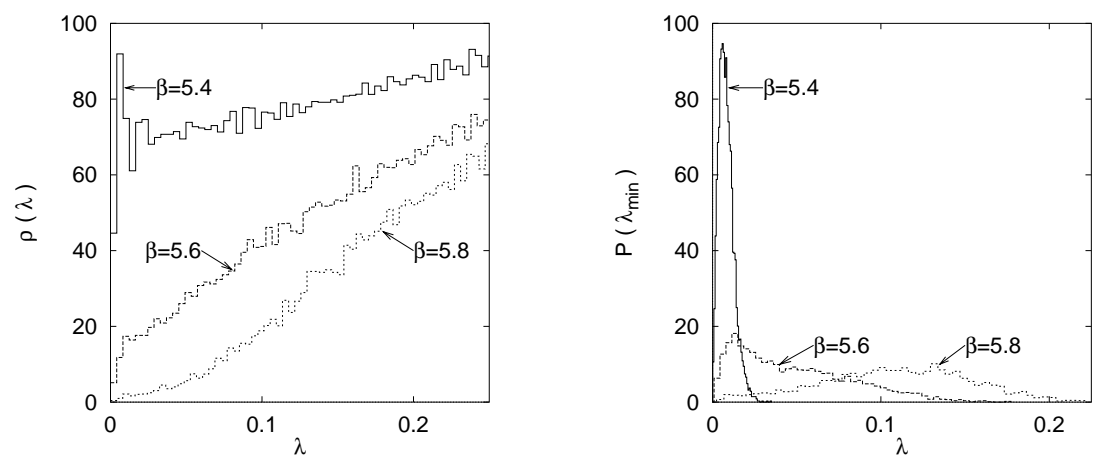

Figure 4. Density $\rho(\lambda)$ of small eigenvalues (left) and distribution $P\left(\lambda_{\min }\right)$ (right) for $\mathrm{SU}(3)$ gauge theory on a $4^{4}$ lattice across the phase transition.
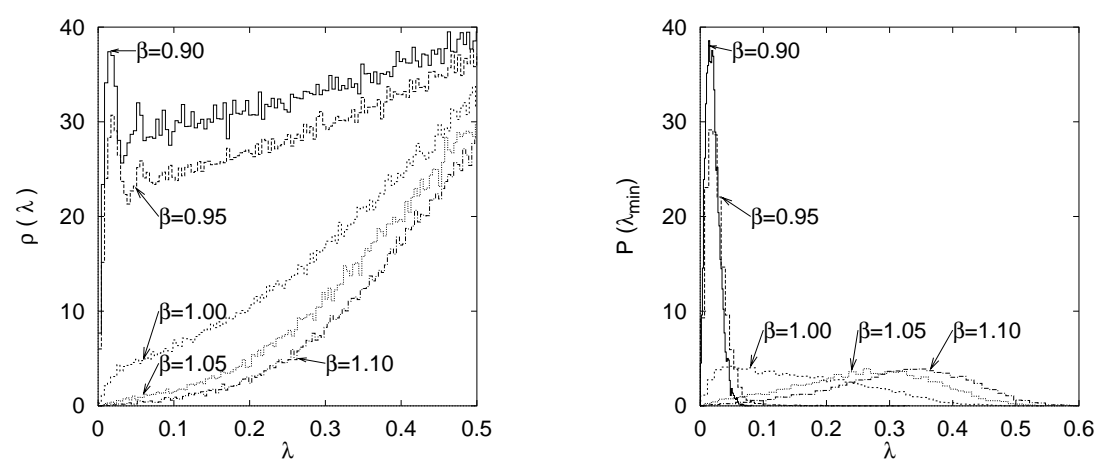

Figure 5. Density $\rho(\lambda)$ of small eigenvalues (left) and distribution $P\left(\lambda_{\min }\right)$ (right) for $\mathrm{U}(1)$ gauge theory on a $4^{4}$ lattice across the phase transition.


Figure 6. Fit of the spectral density to $\rho(\lambda)=C\left(\lambda-\lambda_{0}\right)^{2 m+1 / 2}$ in SU(3) at $\beta=5.8$ (left) and in U(1) at $\beta=1.10$ (right). The contribution of the smallest eigenvalue, the $11^{\text {th }}$ eigenvalue and the $21^{\text {st }}$ eigenvalue is inserted. 
fects should decrease. Further, we checked that the high-end of the spectrum behaves similar to the low-end and thus deviates from the results of the ordinary UE of RMT [17]. Again the question arises whether larger lattices possess spectral edges closer to the microscopic semi-circle universality.

\section{Conclusions}

The aim of this contribution was to work out two different types of universalities inherent in quantum field theories with a covariant derivative and their interpretation in terms of RMT. The first type concerns the bulk of the spectrum of the Dirac operator. The nearest-neighbor spacing distribution $P(s)$ agrees with the RMT result in both the confinement and the deconfinement phase of pure gauge theory and of full $\mathrm{QCD}$, except for extremely large values of $\beta$ where the eigenvalues are known analytically. The nearest-neighbor spacing distribution of $4 \mathrm{~d}$ $\mathrm{U}(1)$ quenched lattice gauge theory is described by the chUE of RMT in both the confinement and the Coulomb phase. Even in the deconfinement phase, gauge fields retain a considerable degree of randomness, which apparently gives rise to quantum chaos in these theories.

The second type of universality concerns the low-lying spectra of the Dirac operators of both QCD and QED. In all cases considered, one finds that in the phase in which chiral symmetry is spontaneously broken the distribution $P\left(\lambda_{\min }\right)$ and the microscopic spectral density $\rho_{s}(z)$ are described by chiral RMT. When chiral symmetry is restored one has to rely on ordinary RMT with the related space-time symmetries, but one finds for our lattice size only universal behavior of the macroscopic density $\rho(\lambda)$.

Acknowledgments: This study was supported in part by FWF project P11456-PHY. We thank B.A. Berg, M.-P. Lombardo, and T. Wettig for collaborations 18 .

\section{REFERENCES}

1. T. Banks and A. Casher, Nucl. Phys. B 169 (1980) 103.

2. M.A. Halasz and J.J.M. Verbaarschot, Phys.
Rev. Lett. 74 (1995) 3920; M.A. Halasz, T. Kalkreuter, and J.J.M. Verbaarschot, Nucl. Phys. B (Proc. Suppl.) 53 (1997) 266.

3. O. Bohigas, M.-J. Giannoni, and C. Schmit, Phys. Rev. Lett. 52 (1984) 1.

4. T.S. Biró, N. Hörmann, H. Markum, and R. Pullirsch, Nucl. Phys. B (Proc. Suppl.) 86 (2000) 403.

5. J.J.M. Verbaarschot, Phys. Rev. Lett. 72 (1994) 2531.

6. D. Fox and P.B. Kahn, Phys. Rev. 134 (1964) B1151; T. Nagao and M. Wadati, J. Phys. Soc. Jpn. 60 (1991) 3298; 61 (1992) 78; 61 (1992) 1910.

7. R. Pullirsch, K. Rabitsch, T. Wettig, and H. Markum, Phys. Lett. B 427 (1998) 119.

8. M.L. Mehta, Random Matrices, 2nd ed. (Academic Press, San Diego, 1991).

9. B.A. Berg and C. Panagiotakopoulos, Phys. Rev. Lett. 52 (1984) 94.

10. B.A. Berg, H. Markum, and R. Pullirsch, Phys. Rev. D 59 (1999) 097504.

11. E.V. Shuryak and J.J.M. Verbaarschot, Nucl. Phys. A 560 (1992) 306; J.J.M. Verbaarschot and I. Zahed, Phys. Rev. Lett. 70 (1993) 3852.

12. H. Leutwyler and A.V. Smilga, Phys. Rev. D 46 (1992) 5607.

13. M. Göckeler, H. Hehl, P.E.L. Rakow, A. Schäfer, and T. Wettig, Phys. Rev. D 59 (1999) 094503.

14. B.A. Berg, H. Markum, R. Pullirsch, and T. Wettig, hep-lat/0007009.

15. M.J. Bowick and E. Brézin, Phys. Lett. B 268 (1991) 21; E. Kanzieper and V. Freilikher, Phys. Rev. E 55 (1997) 3712.

16. F. Farchioni, P. de Forcrand, I. Hip, C.B. Lang, and K. Splittorff, Phys. Rev. D 62 (2000) 014503; P.H. Damgaard, U.M. Heller, R. Niclasen, and K. Rummukainen, Nucl. Phys. B 583 (2000) 347.

17. P.J. Forrester, Nucl. Phys. B 402 (1993) 709.

18. B.A. Berg, H. Markum, R. Pullirsch, and T. Wettig, hep-lat/9912055; B.A. Berg, E. Bittner, M.-P. Lombardo, H. Markum, R. Pullirsch, and T. Wettig, hep-lat/0007008. 